\title{
Fully-Integrated, High-Efficiency, Multi-Output Charge Pump for High-Density Microstimulators
}

\author{
Amin Rashidi ${ }^{1,2}$, Niloofar Yazdani ${ }^{1}$, and Amir M. Sodagar ${ }^{3}$ \\ ${ }^{1}$ Research Laboratory for Integrated Circuits and Systems (ICAS), Faculty of Electrical Engineering, K.N. Toosi \\ University of Technology, Tehran, Iran \\ ${ }^{2}$ Integrated Circuits and Electronics Laboratory (ICELAB), Department of Engineering, Aarhus University, Aarhus, \\ Denmark \\ ${ }^{3}$ Department of Electrical Engineering and Computer Science, York Univerisy, Toronto, ON, Canada \\ E-mail: a.rashidi@eng.au.dk,n.yazdani@eng.au.dk, sodagar@eecs.yorku.ca
}

\begin{abstract}
This paper proposes a high-efficiency charge pump circuit with small integrated capacitors, dedicated to high-density microstimulators. The proposed circuit offers improvement of about $35 \%$ in the charge pump efficiency over the conventional cross-coupled charge pumps. This is achieved through proper employment of two techniques: (a) omitting the undesired conductive paths that discharge the output capacitor, and (b) discounting the dynamic switching power losses by half. Moreover, a straightforward physical layout is proposed to prevent the latchup phenomenon. Occupying $0.5 \mathrm{~mm}^{2}$ of silicon area, circuits for a 4-stage (1 positive stage and 3 negative stages) charge pump were designed and simulated in transistor level in a standard $0.18-\mu \mathrm{m}$ CMOS technology. Designed for an implantable visual prosthesis, the charge pump generates output voltages of $3.48 \mathrm{~V},-1.69 \mathrm{~V}$, $3.38 \mathrm{~V}$, and $-5.05 \mathrm{~V}$ out of a $1.8 \mathrm{~V}$ input voltage and exhibits average power efficiency of $92.8 \%$ and $86.8 \%$ for 1 - and 3-stage circuits respectively, all in the case of a $100 \mu \mathrm{A}$ current load. An output per stage with current sinking/sourcing ability allows different stimulation channels to be independently connected to different supply levels according their operational needs.
\end{abstract}

Keywords-charge pump, charge-sharing; adaptive power supply; current-controlled microstimulator;

\section{INTRODUCTION}

In high-density mirostimulators (HDMS) such as implantable visual prostheses, charge pump (CP) circuits have proved to be successful candidates for providing rather large supply voltage levels to the output stage [1-5]. Fig. 1 presents an overview of an HDMS with the option of recording neural activity. Such an option is usually envisioned to return feedback from the neural response to stimulations [6]. The solid arrows illustrate the data and power distribution required for stimulation and dashed arrows show the flow of the recorded signals to be returned to the outside. Output stage of high-density microstimulators needs to operate with a supply voltage that is usually larger than the supply voltage recommended for today's regular microfabrication technologies (unless more expensive processes with the high-voltage option is used). Thus, a power management block is usually used in such systems in order to convert the regular voltage level $\left(\mathrm{V}_{\mathrm{dd}}\right)$ to higher supply level/levels that are proper for stimulation output stages [2]. Recently, for DC voltage level boosting in microstimulators, the Pelliconi charge pump (PCP) scheme [7] has been preferred over other schemes because of several reasons: fully integrated implementation, compatibility with low-voltage micro-

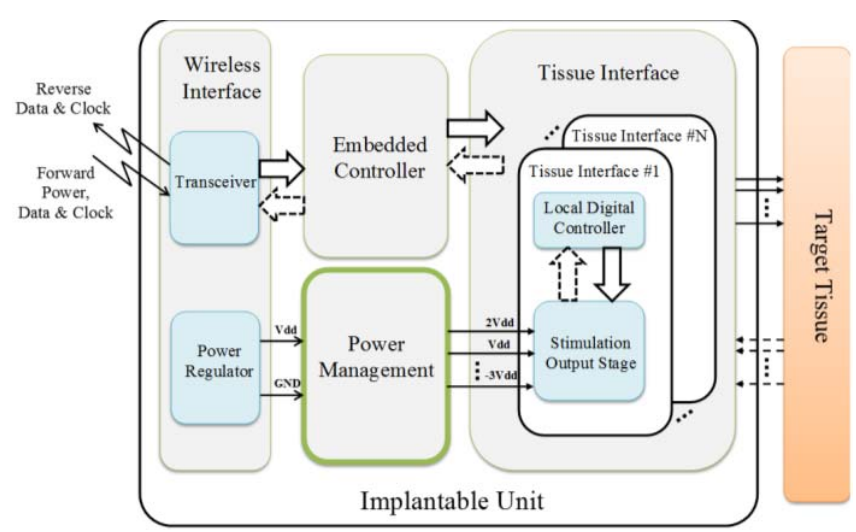

Fig. 1. Block diagram of a high-density microstimulator featuring a power management block with multi-level voltage supplies for adaptive configuration of multiple stimulation channels

fabrication technology, and capability of generating negative voltage levels as well as positive ones [1-5].

In general, there are two main sources of power loss that can potentially result in a not-so-high power efficiency for the PCP: (a) In the majority of fully-integrated CP circuits, the dynamic power loss associated with the parasitic bottom-layer capacitances of pumping capacitors is a major concern [7-9]. For the sake of alleviating switching power loss, charge-sharing between the parasitic bottom-layer capacitors of pumping capacitors was firstly proposed in [8]. This technique is applicable to Dickson, heap, and Fibonacci CPs. (b) Reverse current paths from the output to the input/pumping capacitors results in some output voltage drop and subsequently the degeneration of the overall power efficiency. In order to remove the reverse conductive paths, four-phase charge pumps were proposed with different clock pairs for charge pumping and clock boosting $[4,5,10]$. The four-phase charge pump proposed in [4] adopts a charge-sharing technique to improve the power efficiency. However, as it will be explained later, this technique may result in existence of a reverse current path from the output to the pumping capacitors during the charge-sharing period.

This paper describes the problem with both the conventional PCP and the circuit proposed in [4], and proposes a six-phase charge pump circuit that supports the charge-sharing idea without suffering from the aforementioned reverse current paths. 
The modified PCP proposed in this work addresses the issues of avoiding the latch-up phenomenon [2] and also supporting concurrent stimuli at different supply levels [1].

\section{Proposed Charg Pump Circuit}

\section{A. Power Efficiency}

Primitive cell (the $h^{\text {th }}$ stage) of the basic PCP introduced in [7] is shown in Fig. $2 a$, in which $\phi_{1}$ and $\phi_{2}$ are non-overlapping clock signals, depicted on the right-hand-side. In the steady state, where the pumping capacitors $C_{P U, h}$ and $C_{P D, h}$ are charged to $V_{D D}$, waveforms of the voltages at nodes $P 1$, and $P 2$ are similar to $\phi_{1}$ and $\phi_{2}$, except that both high and low voltage levels are shifted up by $V_{\text {Low. }}$. For a positive CP, $V_{\text {Low }}$ is the input and $V_{\text {High }}$ is the output, and for a negative CP the input and output nodes are swapped. However, it is assumed hereafter that the CP stage under study is a positive one. One of the drawbacks of the basic PCP stage of Fig. $2 a$ is as follows: During $\Delta t$, where both $P 1$ and $P 2$ are low, although both NMOS transistors are turned off, both PMOS devices are on. This provides conductive paths from $V_{\text {High }}$ to nodes $P 1$ and $P 2$ which are both at $V_{\text {Low }}$, allowing for possible discharge of the output node. This results in the degradation of the power efficiency of the CP. Employing different clock pairs for charge pumping and clock boosting can resolve this problem [10]. The timing signals to realize the charge pump function in this scheme are presented in Fig. 2b, where a charge-sharing technique is also employed.
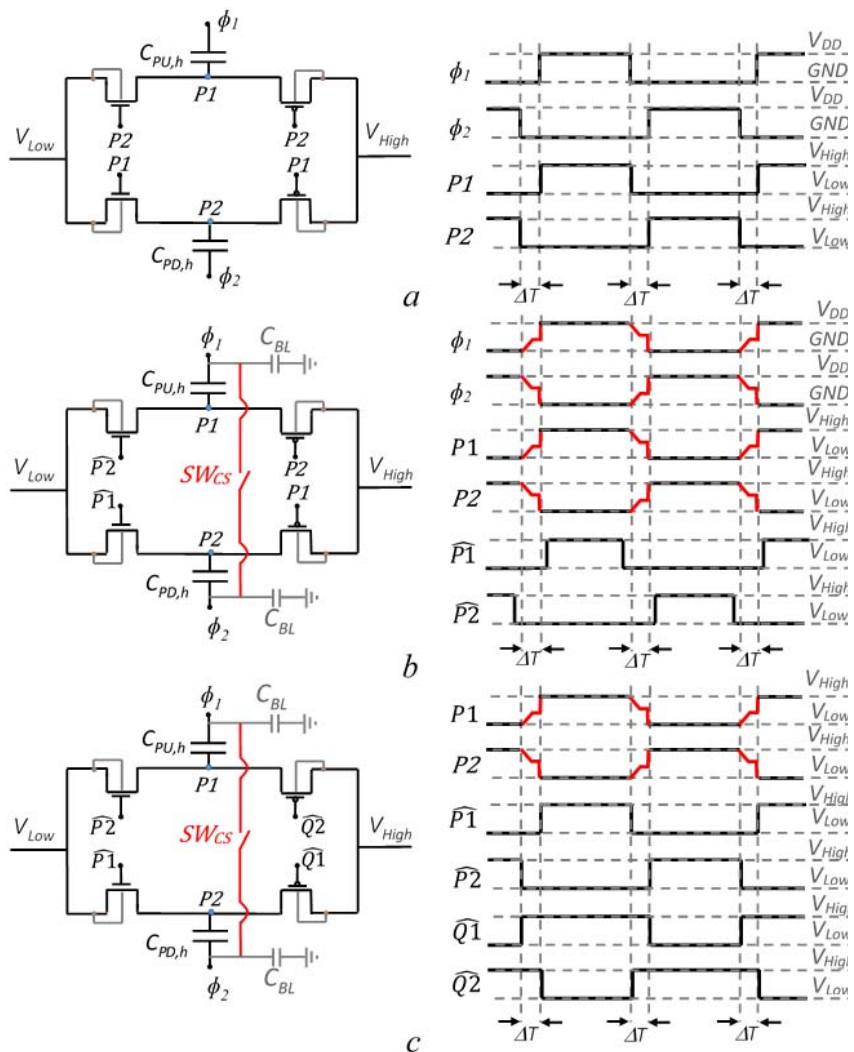

Fig. 2. Timing signals for the cross-coupled charge pump circuits in (a) basic Peliconi (b) four-phase charge pump proposed in [10] with charge-sharing technique (c) Proposed six-phase charge pump
To describe the charge-sharing technique that is used in this work, bottom-layer capacitors $C_{B L}$ associated with the pumping capacitors are drawn in Fig. $2 \mathrm{~b} \& 2 \mathrm{c}$ in grey. During $\Delta t$, one of the $C_{B L}$ capacitors is charged to $V_{D D}$ and the other is fully discharged. The idea here is to connect the two bottom-layer capacitors in parallel using $S W_{C S}$ during $\Delta t$, where the pumping capacitors are to be left unused. As a result, instead of losing all the energy stored in the fully-charged $C_{B L}$, part of its charge is transferred to the other $C_{B L}$. This reduces the energy that the associated clock driver would have to spend on charging the discharged $C_{B L}$ in the following cycle, by half. However, based on the timings shown in Fig. $2 \mathrm{~b}$, during $\Delta t$ the voltage levels at the gates of the PMOS devices $(P 1$ and $P 2)$ are lower than the output voltage $\left(\mathrm{V}_{\text {High }}\right)$. Similar to the basic PCP, this clocking scheme (also used in [4]) results in reverse current flow from the output to the pumping capacitors, and the overall power efficiency is consequently degraded. To solve this problem, three different clock pairs are proposed for the NMOS switches, PMOS switches and for clock boosting. As depicted in Fig. 2c, while the NMOS switches are still controlled using the same non-overlapping signals ( $P 1$ and $P 2$ ), gates of the PMOS switches are connected to proper 'overlapping' signals $(Q 1=\overline{P 1}$ and $Q 2=\overline{P 2}$ ). As a result, during $\Delta t$, all the four switches are turned off as desired, and the charge-sharing between the $\mathrm{C}_{\mathrm{BL}}$ parasitic capacitances does not make any reverse conductive path. To allow for this idea to work in Fig. 2(c), two considerations need to be taken into account:

- During $\Delta \mathrm{t}$, where charge-sharing between the two $\mathrm{C}_{\mathrm{BL}} \mathrm{S}$ takes place, voltages at nodes $P 1$ and $P 2$ will not be appropriate to keep the PMOS/NMOS transistors off. The four signals needed to be applied to the gates of the four transistors will therefore need to be generated by a separate circuit, simplified schematic of which is shown in Fig. 3. To generate $\widehat{P 1}$ and $\widehat{P 2}$, a small-size DC level shifter (which is indeed half of a PCP stage) is used. Then, $\widehat{Q 1}$ and $\widehat{Q 2}$ are generated by simply inverting $\widehat{P 1}$ and $\widehat{P 2}$, as shown in Fig. 3 .

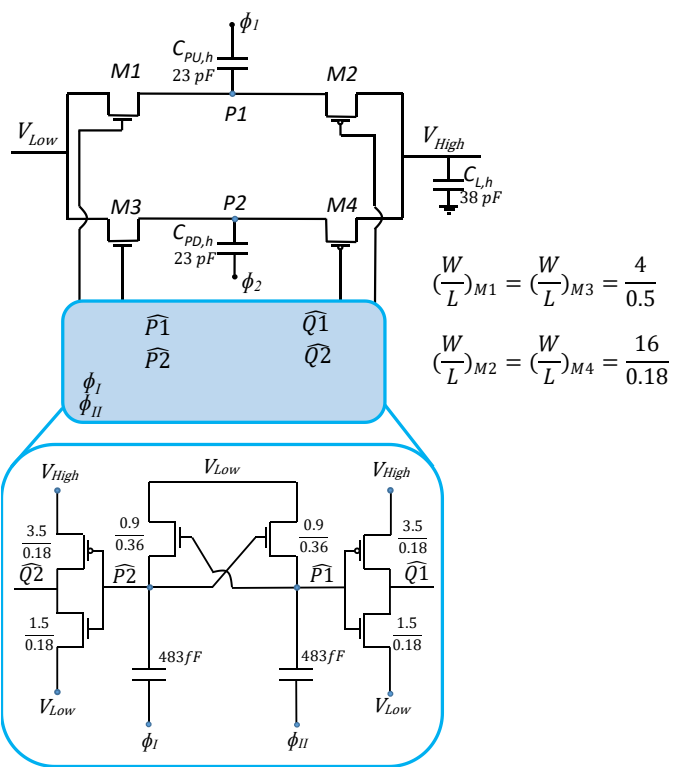

Fig.3. Circuit implementation of the proposed six-phase charg pump 

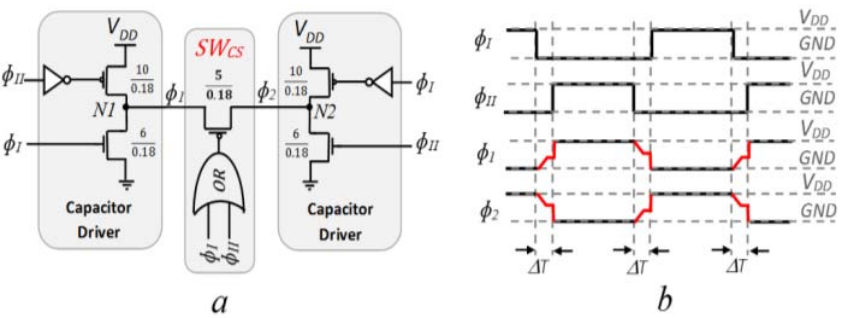

Fig. 4. Redesigned clock driver and its associated input \& output signals

- The clock drivers providing $\phi_{1}$ and $\phi_{2}$ to the CP need to be redesigned in such a way that they exhibit high-impedance outputs during $\Delta t$. Fig. $4(\mathrm{a}) \&(\mathrm{~b})$ present transistor-level schematics of the redesigned clock drivers as well as the associated input and output signals, respectively. This circuit receives two non-overlapping clocks $\phi_{I}$ and $\phi_{I I}$ at the input, generates $\phi_{1}$ and $\phi_{2}$ at the output, and allows for chargesharing between the $\mathrm{C}_{\mathrm{BL}}$ capacitors during $\Delta t$.

\section{B. Supporting Efficient Operation for Concurrent Stimuli}

As depicted in Fig. 3, a 38-pF load capacitor $\left(C_{L}\right)$ is connected from the output of each stage to the ground. This is to allow each output node to operate as a separate and independent voltage supply node. Thus, each channel can select its supply level according to the associated specific voltage/current condition and independently from other channels. This is described thoroughly in [1].

\section{Latch-up Phenomenon}

Conventional PCP is susceptible to the latch-up phenomenon. A common remedy for this problem, proposed in the literature, is the dynamic bulk biasing technique [2 - 4]. To ensure that all the bulk-NWell PN junctions in the circuit are reverse biased, it is proposed in [2] to use a comparator per stage. Fig. $5 a$ presents circuit schematic of the modified PCP stage, equipped with a traditional cross-coupled bulk biasing circuit $(\mathrm{Mbl}$ and $\mathrm{Mb3})$ for NMOS transistors and another one $(\mathrm{Mb} 2$ and $M b 4$ ) for PMOS transistors. Fig. $5 b$ shows cross-sectional view of the physical implementation proposed in this work for the transistors in Fig. 5a. In this scheme, all NMOS transistors are realized in a single P-Well as is usual for deep-NWell transistors. The sidewall N-Well is proposed to be designed large enough to accommodate all PMOS transistors of the circuit (instead of realizing PMOS transistors in a separate NWell, which needs to be away from the sidewall NWell of NMOS transistors by some clearance). As shown in Fig. 5c, bulks of PMOS and NMOS transistors $\left(B_{N}\right.$ and $\left.B_{P}\right)$ in this scheme are connected using a

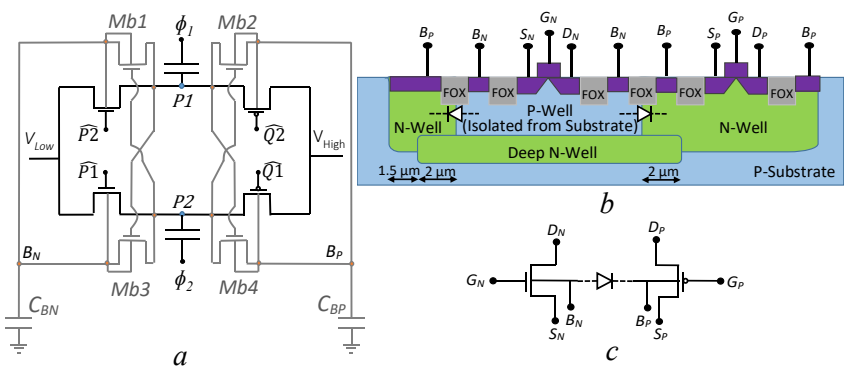

Fig. 5. Dinamic bulk biasing for NMOS and PMOS devices and proposed physical layout to prevent latch-up

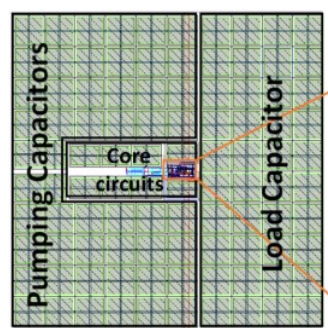

Deep $\mathrm{N}$-well devices in a single P-Well \& PMOS devices on its sidewall $\mathrm{N}$-Well

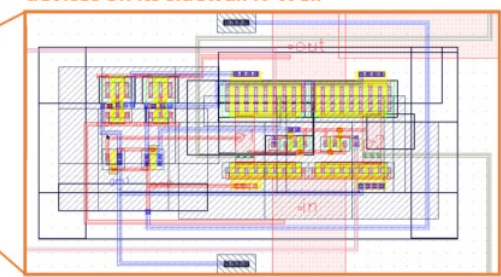

Fig. 6. Physical layout for one stage of the charge pump circuit

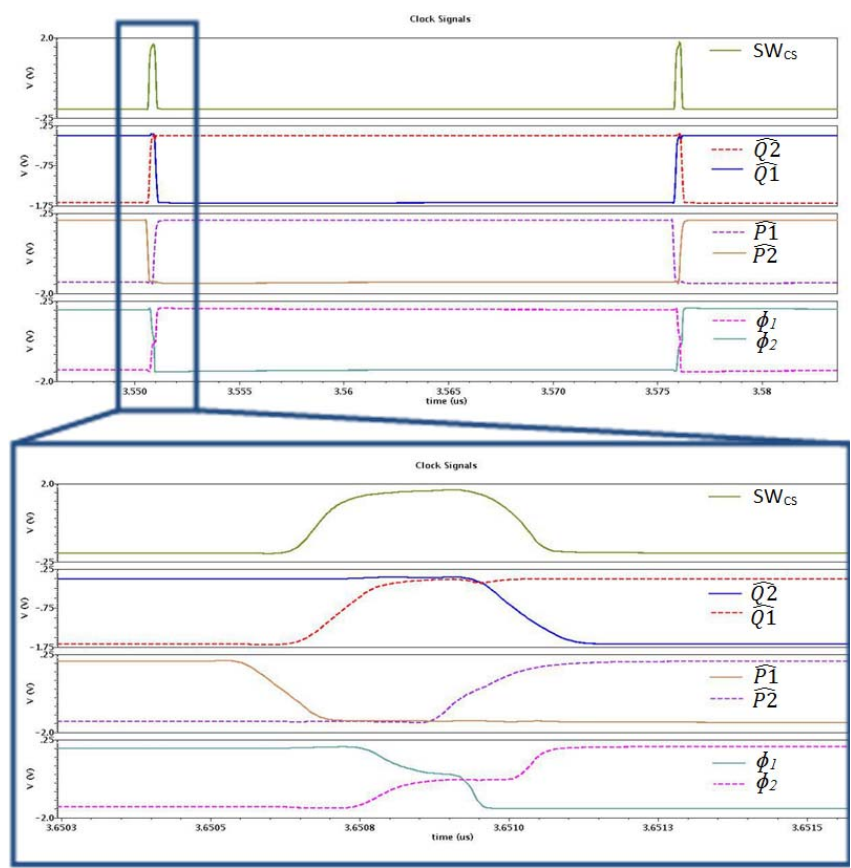

Fig. 7. Simulated timing signals for the first negative stage of the proposed circuit

distributed parasitic diode. Since the dynamic bulk biasing circuit for each CP stage keeps bulks of the associated NMOS transistors always at the lowest possible voltage level and those of the PMOS transistors at the highest possible voltage level, it is guaranteed that in the proposed physical implementation scheme the bulk-NWell PN junctions always remain reverse biased.

\section{POST-LAYOUt SimUlation Results}

Based on the proposed ideas, circuits for 1-stage positive and 3 -stage negative $\mathrm{CP}$ circuits were designed and simulated in transistor level in a standard $0.18 \mu \mathrm{m}$ CMOS technology. Fig. 6 illustrates the physical layout of one stage of the proposed $\mathrm{CP}$, which occupies $345 \mu \mathrm{m} \times 350 \mu \mathrm{m}$ of silicon area. Load capacitor, pumping capacitor, and core circuits consume $40.6 \%, 7.2 \%$, and $50.7 \%$ of the total area, respectively. Fig. 7 shows the simulated timing signals for the first negetive stage of the proposed circuit at a clock frequency of $20 \mathrm{MHz}$. Fig. 8 depicts output voltages of the proposed $\mathrm{CP}$ circuit at the same frequency and with a current load of $100 \mu \mathrm{A}$ connected to the circuit at $\mathrm{t}=2 \mu \mathrm{Sec}$. The voltages $\mathrm{VO}+1$, VO-1, VO-2, and VO-3 in Fig. 8 denote the output voltages of the positive, first negative, second negative, and third negative stages, respectively. At $20 \mathrm{MHz}$ and with a 


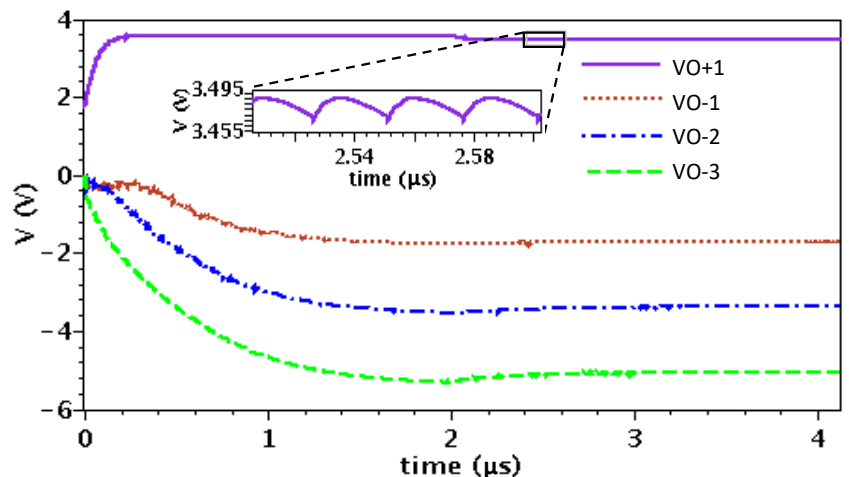

Fig. 8. Outputs of the proposed charge pump circuit

current load of $100 \mu \mathrm{A}$, the proposed CP circuit provides $3.48 \mathrm{~V}$, $-1.69 \mathrm{~V},-3.38 \mathrm{~V},-5.05 \mathrm{~V}$ at the output with average ripple of $22 \mathrm{mV}$ and exhibits power convergence efficiency (PCE) of $92.8 \%$ and $86.8 \%$ for 1 - and 3-stage circuits respectively. Specifications of the proposed CP resulted from post-layout simulations are summarized in Table I.

\section{CONCLUSION}

A multi-output charge pump circuit dedicated to highdensity microstimulators is reported. The proposed six-phase charge pump circuit benefits from a charge-sharing technique that is envisioned for dynamic power loss reduction. In the proposed circuit, the reverse conductive paths are removed, resulting in the achievement of a high power efficiency. Physical layout of the circuit suggests a well-thought design for preventing the latch-up phenomenon to happen. This is achieved through taking advantage of the different semiconductor layers readily available in the deep $\mathrm{N}-$ Well CMOS process.

\section{REFERENCES}

[1] A. Rashidi, N. Yazdani and A. Sodagar, "Fully-implantable, multichannel microstimulator with tracking supply ribbon and energy recovery," 38th Anual International Conference of IEEE EMBS, pp. 18181821, Orlando, USA, 2016.

[2] M. H. Maghami, A. M. Sodagar and M. Sawan, "Versatile stimulation back-end with programmable exponential current pulse shapes for a retinal visual prosthesis," IEEE Transaction on Neural Systems and Rehabilation Engineering, vol. 24, no. 11, pp. 1243-1253, 2016.

[3] S. Ethier and M. Sawan, "Exponential current pulse generation for efficient very high-impedance multisite stimulation," IEEE Transaction on Biomedical Circuits and Systems, vol. 5, no. 1, pp. 30-38, 2011.

[4] Z. Luo and M.-D. Ker, "A high-voltage-tolerant and power-efficient stimulator with adaptive power supply realized in low-voltage CMOS process for implantable biomedical applications," IEEE Journal on Emerging and Selected Topics in Circuits and Systems, vol. 8, no. 2, pp. 178-186, 2018 .
Table I: Summary of specifications and comparison

\begin{tabular}{|c|c|c|c|c|}
\hline Specification & $\begin{array}{c}\text { JSSC } 2003 \\
{[7]}\end{array}$ & $\begin{array}{l}\text { TNSRE } \\
\text { 2016[2] }\end{array}$ & $\begin{array}{c}\text { TBIOCAS } \\
2017 \text { [5] }\end{array}$ & $\begin{array}{c}\text { This } \\
\text { Work }\end{array}$ \\
\hline $\begin{array}{c}\text { Max. PCE } \\
(\%)\end{array}$ & 50 & $\begin{array}{l}\text { Pos. } 60, \\
\text { Neg. } 48\end{array}$ & $69 \%$ & $\begin{array}{l}\text { Pos. 92.8, } \\
\text { Neg. 86.8 }\end{array}$ \\
\hline $\begin{array}{c}\text { Voltage } \\
\text { convergence } \\
\text { ratio }(\mathrm{V} / \mathrm{V})\end{array}$ & $7 / 1.8$ & $\begin{array}{c}(3.08,-3) / \\
1.2\end{array}$ & $11.3 / 3.3$ & $\begin{array}{c}(3.38,- \\
5.05) / 1.8\end{array}$ \\
\hline $\begin{array}{c}\text { Num. of } \\
\text { Stages }\end{array}$ & 5 pos. & $\begin{array}{c}2 \text { Pos., } 3 \\
\text { Neg. }\end{array}$ & 3 & $\begin{array}{c}1 \text { Pos., } 3 \\
\text { Neg. }\end{array}$ \\
\hline $\begin{array}{l}\text { DC current } \\
\text { load }(\mu \mathrm{A})\end{array}$ & 35 & 200 & 500 & 100 \\
\hline $\begin{array}{l}\text { Output ripple } \\
\text { (PP) }\end{array}$ & $400 \mathrm{mV}$ & $1.3 \%$ & $2.6 \%$ & $22 \mathrm{mV}$ \\
\hline $\begin{array}{c}\text { Capacitance } \\
(\mathrm{pF})\end{array}$ & 55 & 690 & 400 & 350 \\
\hline $\begin{array}{l}\text { Off-chip } \\
\text { capacitor }\end{array}$ & No & No & $1-\mathrm{nF}$ & No \\
\hline $\begin{array}{c}\text { Clock freq. } \\
(\mathrm{MHz})\end{array}$ & 0.1 & 8 & $7-50$ & 20 \\
\hline $\begin{array}{c}\text { Silicon area } \\
\left(\mathrm{mm}^{2}\right)\end{array}$ & $\mathrm{NA}$ & 0.5 & $\mathrm{NA}$ & 0.5 \\
\hline $\begin{array}{l}\text { Technology } \\
(\mu \mathrm{m})\end{array}$ & 0.18 & 0.13 & 0.18 & 0.18 \\
\hline $\begin{array}{l}\text { Multi-output } \\
\text { CP }\end{array}$ & No & No & No & Yes \\
\hline $\begin{array}{l}\text { Simulation/ } \\
\text { experimental } \\
\text { results }\end{array}$ & experimental & experimental & experimental & simulation \\
\hline
\end{tabular}

[5] Z. Luo, M.-D. Ker, T.-Y. Yang and W.-H. Cheng, "A digitally dynamic power supply technique for 16-channel $12 \mathrm{~V}$-tolerant stimulator realized in a $0.18-\mu \mathrm{m} \quad 1.8-\mathrm{V} / 3.3-\mathrm{V}$ low-voltage CMOS process," IEEE Transaction on Biomedical Circuits and Systems, vol. 11, no. 5, pp. 10871096, 2017.

[6] H. Chandrakumar, and D. Marković, "A high dynamic-range neural recording chopper amplifier for simultaneous neural recording and stimulation," IEEE Journal of Solid State Circuits, vol. 52, no. 3, pp. 645656,2017

[7] R. Pelliconi, D. Iezzi, A. Baroni, M. Pasotti and P. Rolandi, "Power efficient charge pump in deep submicron standard CMOS technology," IEEE Journal of Solid State Circuits, vol. 38, no. 6, pp. 1068-1071, 2003.

[8] Y. Allasasmeh and S. Gregori, "Charge reusing in switched-capacitor voltagemultipliers with reduced dynamic losses," 53rd IEEE International Midwest Symposium on Circuits and Systems, pp. 11691172, Seattle, USA, 2010.

[9] G. Palumbo, and D. Pappalardo, "Charge pump circuits: An overview on design strategies and topologies," IEEE Circuits and Systems Magasin, vol 10, no. 1 , pp. $31-45,2010$.

[10] R. Karadi and G. V. Pique, "3-phase 6/1 switched-capacitor DC-DC boost converter providing $16 \mathrm{~V}$ at $7 \mathrm{~mA}$ and $70.3 \%$ efficiency in $1.1 \mathrm{~mm} 3$," Proceeding of IEEE International Solid-State Circuits Conference, pp. 92-94, 2014. 